\title{
Biglobal linear stability analysis for the flow in eccentric annular channels and a related geometry
}

\author{
Elia Merzari, ${ }^{1, a)}$ Sheng Wang, ${ }^{1}$ Hisashi Ninokata, ${ }^{1}$ and Vassilis Theofilis ${ }^{2, b)}$ \\ ${ }^{1}$ Research Laboratory for Nuclear Reactors, Tokyo Institute of Technology, N1-5, 2-12-1 O-okayama, \\ Meguro-ku, Tokyo 152-8550, Japan \\ ${ }^{2}$ School of Aeronautics, Universidad Politécnica de Madrid, Plaza Cardenal Cisneros 3, \\ 28040 Madrid, Spain
}

(Received 22 March 2008; accepted 16 September 2008; published online 25 November 2008)

\begin{abstract}
Recently, it has been observed that simple geometry characterized by a low level of symmetry present interesting peculiarities in the process of transition from laminar Poiseuille flow to turbulent flow. Examples of this type of geometry are eccentric channels and, more generally, parallel channels containing a narrow gap. In the present work, a global linear stability analysis for the flow in this class of geometry has been performed. The problem is discretized through spectral collocation and the eigenvalue problem has been solved with the Arnoldi-method based algorithms and the QZ algorithm. Since no numerical studies of this type have yet been performed to address the issue of transition in this geometry, the codes have been validated toward results obtained in simplified geometries (e.g., concentric annular channel and square channel). The eigenvalue spectra of the Poiseuille flow in eccentric channels and a U-shaped channel have then been computed and analyzed for a wide range of geometric parameters. After comparison with spectra typical of channel flow and pipe flow it is shown that an additional linear mechanism of instability is present, related to the spanwise variation of the laminar velocity profile. (C) 2008 American Institute of Physics.
\end{abstract}

[DOI: 10.1063/1.3005864]

\section{INTRODUCTION}

The turbulent flow in parallel channels characterized by the presence of a narrow gap is subject to local laminarization, and more generally, turbulence is anisotropic and inhomogeneous in the cross section. Moreover, large-scale periodic coherent structures are usually observed in the region of the narrow gap. ${ }^{1}$ These coherent structures are observed even at very low Reynolds numbers, and appear to be present at an early stage in the process of laminar-turbulent transition for this class of geometries. ${ }^{1}$

No numerical studies regarding the issue of transition in these geometries have been performed. In the present work the focus will be on a biglobal linear stability analysis ${ }^{2}$ of the laminar Pouseille flow in two geometries characterized by the presence of a narrow gap. No such study has been published previously, to the knowledge of the present authors. By biglobal linear stability analysis is intended here a method that does not rely on the approximations of spatial homogeneity in two or three spatial directions, as the OrrSommersfeld theory does by using the Squire transformation, but rather addresses the problem as a partial differential equation eigenvalue problem. It considers arbitrary dependence of the amplitude function on two spatial directions, only assuming periodicity on the third.

The prototype of any geometry containing a narrow gap is the eccentric annulus. This geometry has been studied through a spectral collocation discretization ${ }^{3}$ method written

\footnotetext{
${ }^{a)}$ Author to whom correspondence should be addressed. Telephone and fax: +81-3-5734-3056. Electronic mail: merzari@nr.titech.ac.jp.

b) Telephone: +3491336 3291. Fax: +34913366371.
}

in bipolar coordinates. It has to be noted that the oscillations observed by Guellouz and Tavoularis ${ }^{4}$ and Hopper and Rehme $^{5}$ have not been observed experimentally for eccentric channels. A recent numerical work ${ }^{6}$ supports the assumption that the oscillations are indeed present for the flow in eccentric annuli for certain combinations of the geometric parameters and the Reynolds number.

Since the geometry of Guellouz and Tavoularis ${ }^{4}$ and Gosset and Tavoularis ${ }^{1}$ cannot be analyzed easily due to the absence of a set of orthogonal coordinates, in the present work another geometry has been analyzed. Lexmond et al., ${ }^{7}$ Biemuller et al., ${ }^{8}$ and Meyer and Rehme ${ }^{9}$ showed that then parallel flow in two rectangular channels connected by a narrow gap presents the same behavior observed by Guellouz and Tavoularis ${ }^{4}$ in the region of the narrow gap. The linear stability problem for the laminar flow in this geometry has been analyzed here through a multiblock spectral collocation methodology in Cartesian coordinates.

Figure 1 shows an outline of the geometry studied as well as the laminar velocity profile examined. Figure 2 shows the grids used for the spectral collocation discretization employed. The algorithms used here have been implemented in MATLAB for testing and in FORTRAN for large-scale calculations. Two pre-existing QZ algorithms ${ }^{10}$ have been used (implemented, respectively, in the IMSL library and MATLAB). An in-house implicitly restarted Arnoldi algorithm $^{11}$ implemented in FORTRAN has also been used. Due to the memory requirements all calculations have been performed on the Japanese supercomputer TSUBAME. 

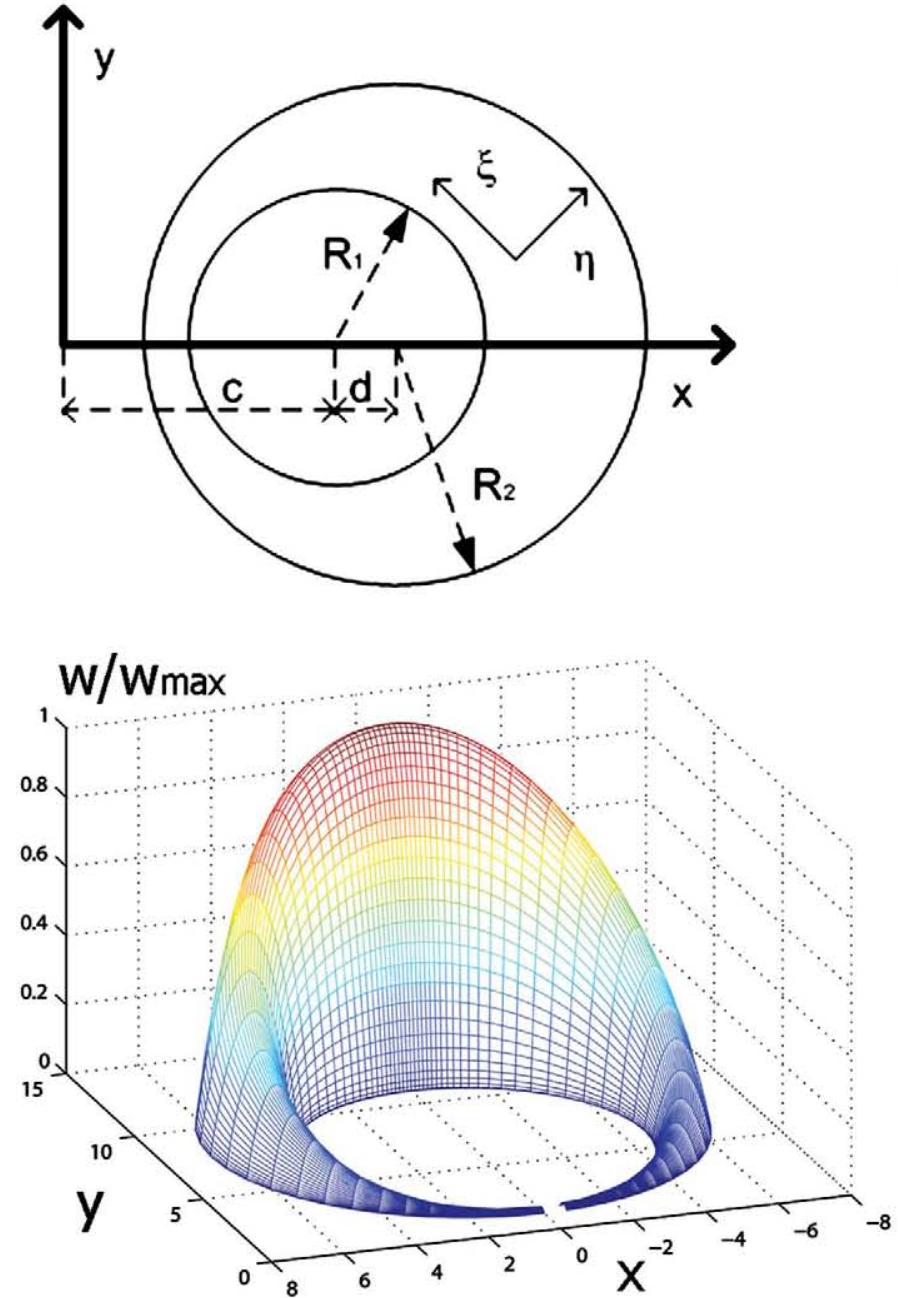
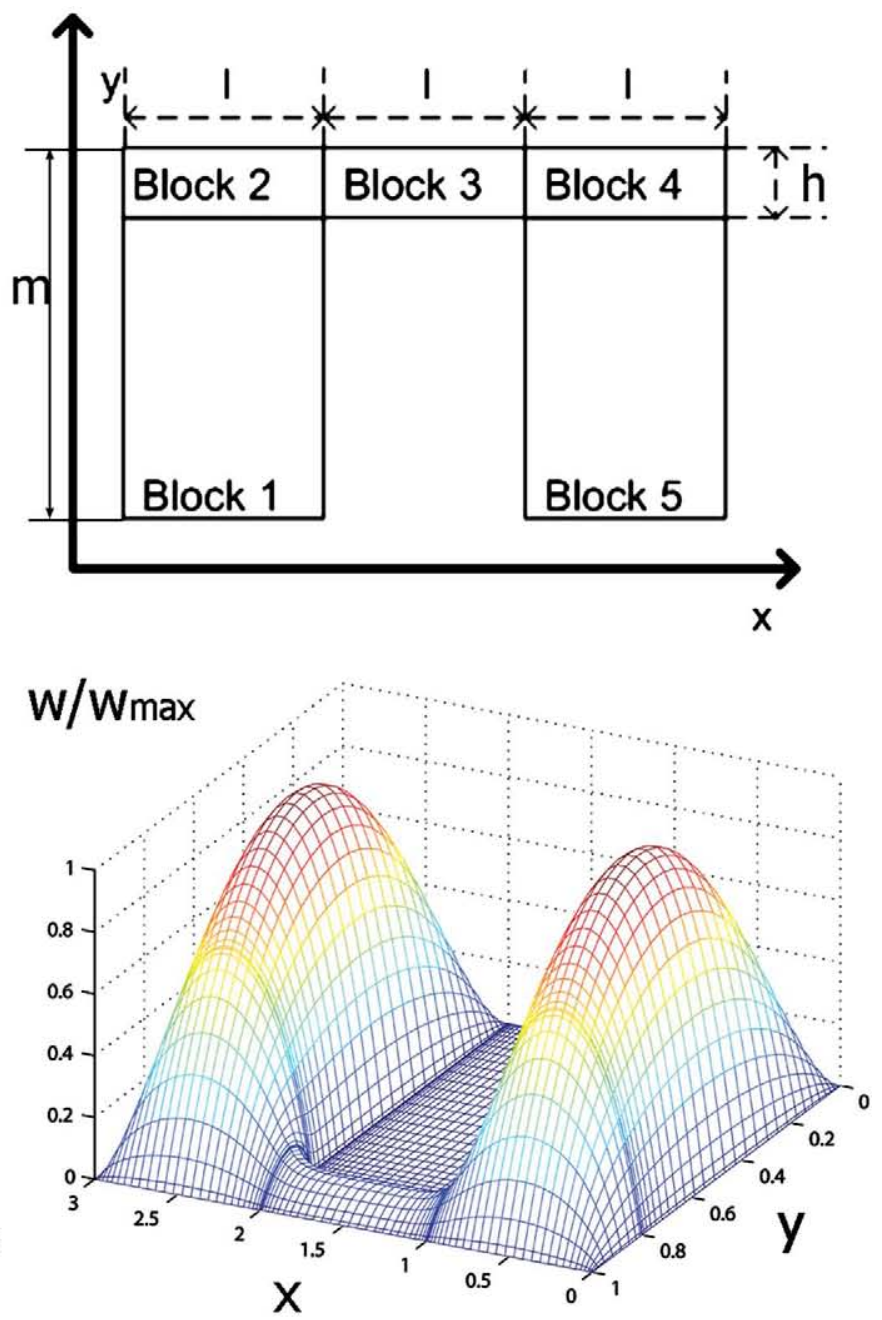

FIG. 1. (Color online) Geometries studied and streamwise velocity laminar profiles.

\section{RECTANGULAR “U SHAPED” CHANNELS}

\section{A. Outline}

The flow in rectangular channels connected by a narrow gap presents flow oscillations in the narrow gap when the narrow gap length to width ratio is bigger than two. The linear stability problem in Cartesian coordinates is expressed by the following eigenvalue-eigenfunction problem for $(\widetilde{u}, \widetilde{v}, \widetilde{w}, \widetilde{p})$ and $\sigma$ (where $z$ is streamwise direction and w the streamwise velocity):

$$
-i \sigma \widetilde{u}=-i W \alpha \tilde{u}-\frac{\partial \widetilde{p}}{\partial x}+\frac{1}{\operatorname{Re}}\left(\frac{\partial^{2} \widetilde{u}}{\partial x^{2}}+\frac{\partial^{2} \tilde{u}}{\partial y^{2}}-\alpha^{2} \tilde{u}\right)
$$
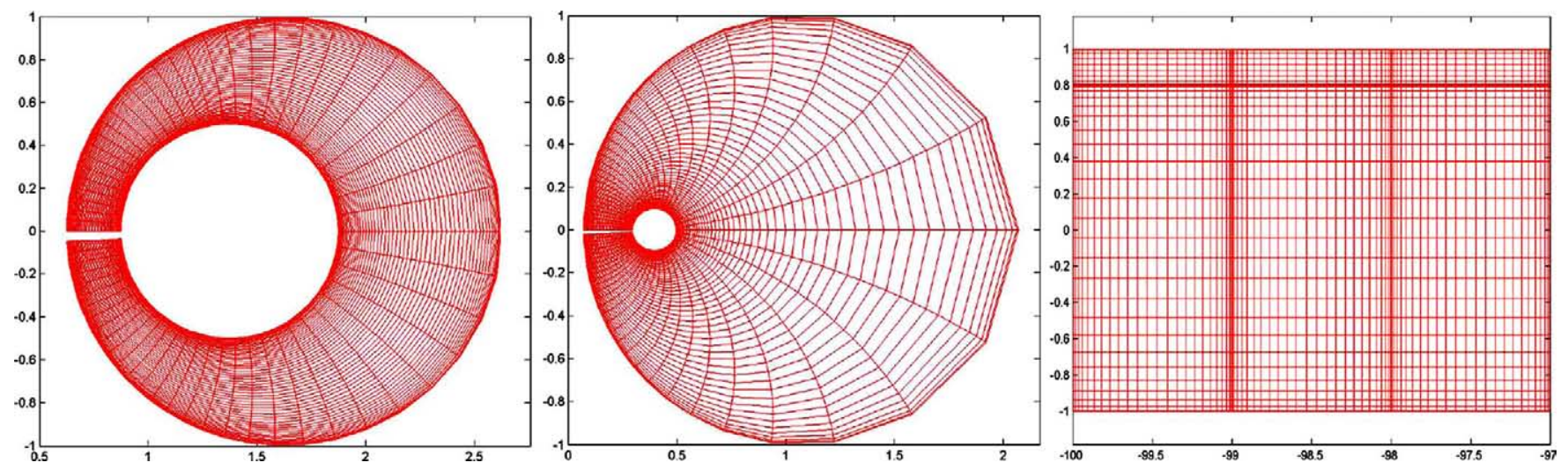

FIG. 2. (Color online) Grids employed. 


$$
\begin{gathered}
-i \sigma \tilde{v}=-i W \alpha \tilde{v}-\frac{\partial \tilde{p}}{\partial y}+\frac{1}{\operatorname{Re}}\left(\frac{\partial^{2} \tilde{v}}{\partial x^{2}}+\frac{\partial^{2} \tilde{v}}{\partial y^{2}}-\alpha^{2} \tilde{v}\right), \\
-i \sigma \tilde{w}=-i W \alpha \tilde{w}-\frac{\partial W}{\partial x} \tilde{u}-\frac{\partial W}{\partial y} \tilde{v}-i \alpha \tilde{p} \\
+\frac{1}{\operatorname{Re}}\left(\frac{\partial^{2} \tilde{w}}{\partial x^{2}}+\frac{\partial^{2} \tilde{w}}{\partial y^{2}}-\alpha^{2} \tilde{w}\right), \\
\frac{\partial \tilde{u}}{\partial x}+\frac{\partial \tilde{v}}{\partial y}+i \alpha \tilde{w}=0
\end{gathered}
$$

where $W$ is the laminar streamwise velocity distribution. The boundary conditions are used for the pressure:

$$
\frac{\partial \tilde{p}}{\partial y}=\frac{1}{\operatorname{Re}}\left(\frac{\partial^{2} \tilde{v}}{\partial y^{2}}\right),
$$

if the wall is normal to the coordinate $y$;

$$
\frac{\partial \widetilde{p}}{\partial x}=\frac{1}{\operatorname{Re}}\left(\frac{\partial^{2} \tilde{u}}{\partial x^{2}}\right),
$$

if the wall is normal to the coordinate $x$; and nonslip boundary conditions for the velocity components. The eigenfunctions $(\tilde{u}, \widetilde{v}, \widetilde{w}, \widetilde{p})$ refer to the perturbations:

$$
\begin{aligned}
u^{\prime} & =\widetilde{u}(x, y) \exp [i(\alpha z-\sigma t)], \\
v^{\prime} & =\widetilde{v}(x, y) \exp [i(\alpha z-\sigma t)], \\
w^{\prime} & =\widetilde{w}(x, y) \exp [i(\alpha z-\sigma t)], \\
p^{\prime} & =\tilde{p}(x, y) \exp [i(\alpha z-\sigma t)] .
\end{aligned}
$$

An additional point of complexity is that the domain has a U-shape and special care needs to be taken to treat this geometry with a spectral collocation method. The cross section has been divided in five blocks, in which both directions have been discretized with Chebyshev polynomials. At the junctions between the blocks the following conditions have been enforced (an example on the boundary between block 1 and block 2):

$$
\begin{aligned}
& \left(\tilde{u}_{1}, \tilde{v}_{1}, \tilde{w}_{1}\right)=\left(\tilde{u}_{2}, \tilde{v}_{2}, \tilde{w}_{2}\right), \\
& \left(\vec{\nabla} \cdot\left(\tilde{u}_{1}, \tilde{v}_{1}, \widetilde{w}_{1}\right)\right) \cdot \vec{n}_{1}=\left(\vec{\nabla} \cdot\left(\tilde{u}_{2}, \tilde{v}_{2}, \tilde{w}_{2}\right)\right) \cdot \vec{n}_{2},
\end{aligned}
$$

where $\vec{n}_{1}$ and $\vec{n}_{2}$ are the vectors normal to the interface between blocks 1 and $2 ;\left(\widetilde{u}_{1}, \widetilde{v}_{1}, \widetilde{w}_{1}\right)$ is the eigenfunction for block 1 and $\left(\tilde{u}_{2}, \tilde{v}_{2}, \tilde{w}_{2}\right)$ the eigenfunction for block 2 . This ensures the well posedness of the linear stability problem.

The laminar solution $W$ has been computed numerically by solving the following problem:

$$
\left(\frac{\partial^{2} W}{\partial x^{2}}+\frac{\partial^{2} W}{\partial y^{2}}\right)=\operatorname{Re} \frac{d P}{d z}
$$

and normalized by the value of the maximum velocity $(d P / d z$ is the mean pressure gradient in the streamwise direction). The grid used is similar in structure to the one used for the eigenvalue problem. However, as shown by Theofilis et al., ${ }^{12}$ the solution of the Poisson equation should take place on a finer grid than the eigenvalue problem mesh. Therefore, Eq. (4) has been solved on a mesh $2 N \times 2 M$ where $M \times N$ is the mesh used for the eigenvalue problem given by Eqs. (1a)-(1d). Once the laminar solution is interpolated on the grid, Eqs. (1a)-(1d) can be reduced to the following linear eigenvalue problem in the temporal framework (obtained considering $\alpha$ as a parameter, searching for the eigenvalue $\sigma$ ):

$$
\overline{\bar{A}} \bar{u}=\sigma \overline{\bar{B}} \bar{u}
$$

where $\bar{u}$ represents a unidimensional vector representation of $(\tilde{u}, \widetilde{v}, \widetilde{w})$, and $\overline{\bar{A}}$ and $\overline{\bar{B}}$ are the matrix associated to the set of equations. The artificial-compressibility paradigm can be applied to avoid matrix $B$ to become singular.

Equation (5) has then been solved with the QZ algorithm $^{10}$ for testing and an Arnoldi algorithm ${ }^{11}$ for largescale computations. In the case of the Arnoldi algorithm the following equivalent eigenvalue problem has been solved:

$$
(\overline{\bar{A}}-\tau \overline{\bar{B}})^{-1} \overline{\bar{B}} \bar{u}=\frac{1}{(\sigma-\tau)} \bar{u},
$$

since it leads to the same set of eigenvalues, shifted by the constant $\tau$, as shown by Jennings and Halliday. ${ }^{13}$ The matrix $(\overline{\bar{A}}-\tau \overline{\bar{B}})$ has been inverted by solving the equation:

$$
(\overline{\bar{A}}-\tau \overline{\bar{B}}) M=\stackrel{\bar{B}}{ },
$$

where $M=(\overline{\bar{A}}-\tau \overline{\bar{B}})^{-1} \overline{\bar{B}}$. The equation has been solved serially (or, in some cases, parallelly with shared memory) with an iterative method (biconjugate gradient method) written in FORTRAN and LU decomposition based methods with MATLAB.

\section{B. Validation}

The validity of the methodology has been tested on the flow in rectangular channels and on the limit of channel flow.

Let $A$ be the ratio between the extension of the domain in the direction $x$ and the extension of the domain in direction $y$. For $A \rightarrow \infty$ the eigenvalue spectrum should approximate the spectrum of the flow between two parallel infinite plates extending in the $x$ direction.

Figure 3 shows results for $A=20$ and $A=200$, compared to the spectrum of channel flow at $\operatorname{Re}=100$. It is possible to notice that the Cartesian bidimensional code is able to reproduce well the spectrum of channel flow. Both results are obtained with a $32 \times 48$ grid. The algorithm used is the QZ algorithm. It is possible to notice that the results are in general good agreement with those presented in Theofilis et al. ${ }^{12}$

\section{Results}

For the geometry of Lexmond et al. ${ }^{7}$ the typical eigenvalue spectrum is shown in Fig. 4. Assuming the geometry to be defined as in Fig. 1, two geometric parameters affect the eigenvalue spectrum: the channel length $L$ and the gap height $H$ (the coordinates have been normalized by the channel height $M$ ). For $L=1 / 3$ and $H=1 / 5$ the flow presents an unstable mode, while for a rectangular geometry with the same 

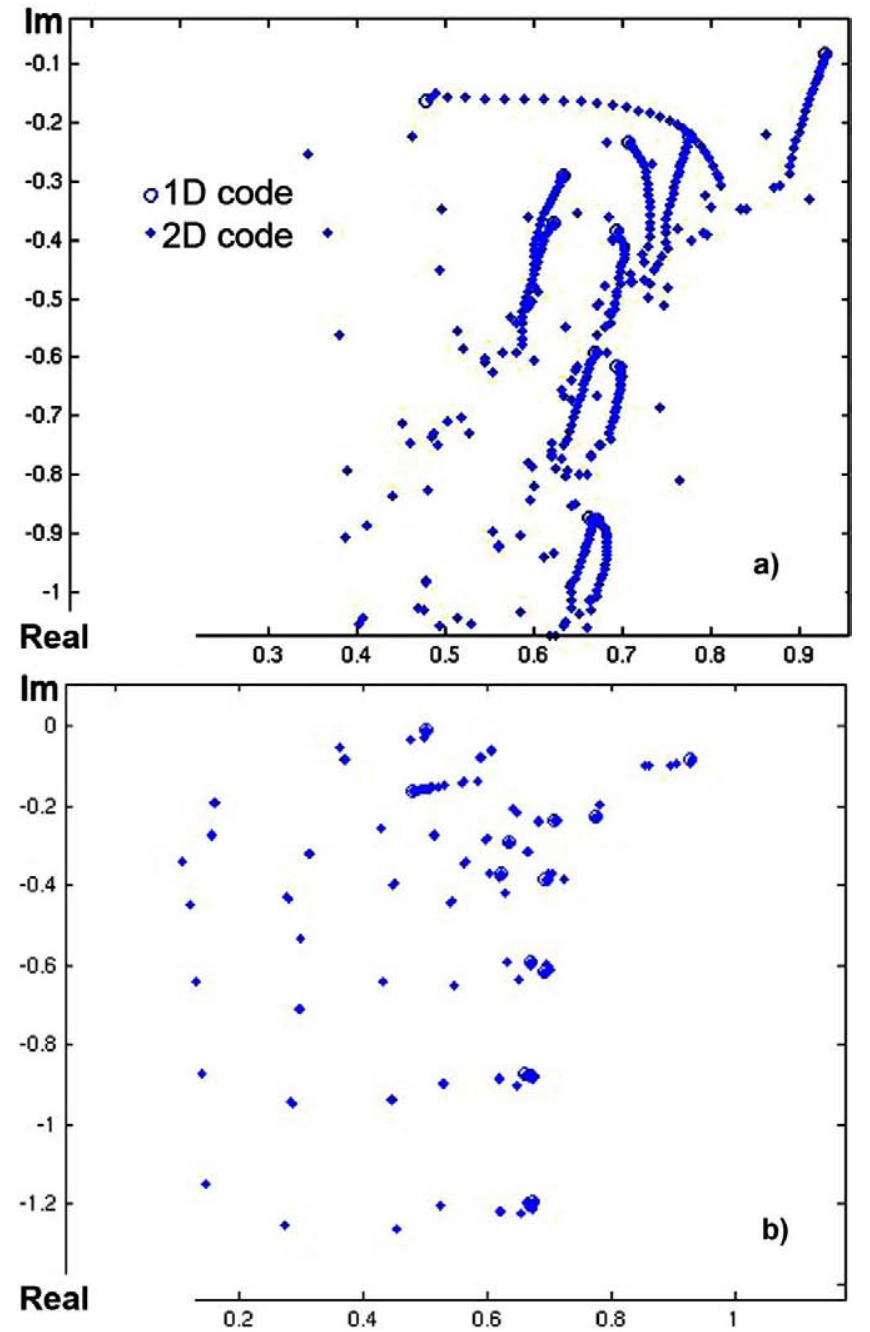

FIG. 3. (Color online) Benchmarking of the 2D multiblock code. Square duct at $\operatorname{Re}=100$ for $A=20$ (a) and $A=200$ (b).

parameter $A(=M / L=3)$ the flow is linearly stable for every value of Re. A convergence study on the most unstable eigenmode has been conducted, and the results are reported in Table I.

The most unstable mode is characterized by an antisymmetric pressure wave (Fig. 5) propagating in the streamwise direction. This wave is associated with a strong oscillating cross velocity in the narrow gap region. A similar mechanism of instability will be observed in the following section for eccentric channels, where its origin will be investigated. The cross velocity and streamwise velocity distributions (Fig. 5) of the most unstable perturbation are strikingly similar to the distributions observed for the most energetic mode of turbulence at a Reynolds number of 3000 through proper orthogonal decomposition (POD) [for a large eddy simulation (LES) in the same geometry as shown by Merzari et al. $\left.{ }^{14}\right]$. This suggests that the laminar velocity profile in this geometry is linearly unstable and the most unstable mode develops into the most energetic mode of turbulence.

For narrow gaps the critical Reynolds number is sensibly lower than the value predicted by the linear theory for the flow in parallel plates and square ducts of any shape (Table

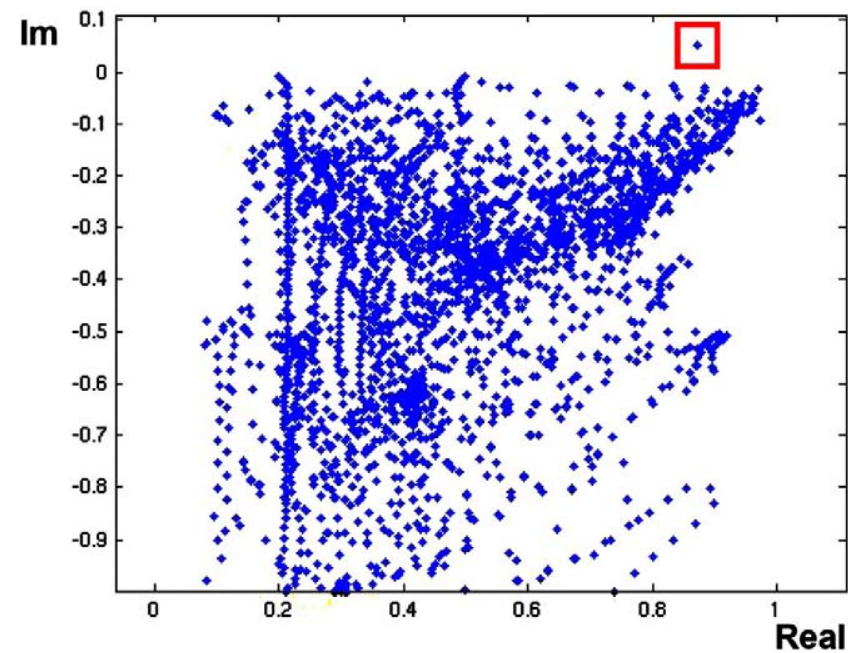

FIG. 4. (Color online) Eigenvalue spectrum $L=1 / 3, H=1 / 5, \operatorname{Re}=50000$, and $\alpha=1.0$.

II). It is also reasonably close to experimental values characteristic of rectangular ducts for the values of $H$ investigated here. ${ }^{2,7}$ As an example, the curves of marginal stability for three different values of the parameter are shown in Fig. 6. It is possible to notice that the position of the minima of the curves strongly depends upon the parameter $L$.

The results presented in this section show clearly that the introduction of a narrow gap changes the stability properties of the flow. In particular two otherwise linearly stable rectangular ducts become unstable when connected through a gap. The origin of this surprising result will be investigated in the following section, by studying a simpler geometry.

\section{ECCENTRIC CHANNELS}

\section{A. Outline}

The linear stability problem for the flow in eccentric channels will be considered. Even if this geometry is simple, and an orthogonal set of coordinates exists, the problem has never been addressed before, to the knowledge of the present authors. The only available case for eccentric channels appears to be the flow between counter-rotating cylinders, widely studied experimentally and theoretically by Taylor ${ }^{15}$ and object of several analytical and numerical studies. ${ }^{16}$ However the conditions of instability for Taylor-Couette flows are different from the ones in the present case and no direct comparison is possible.

TABLE I. Most unstable eigenvalues for $L=0.33, H=0.2 \quad e=0.5$, $\operatorname{Re}=50000, \alpha=1$.

\begin{tabular}{cc}
\hline \hline Grid $\left(\mathbf{N}_{\mathbf{x}} \times \mathbf{N}_{\mathbf{y}}\right)$ & Most unstable \\
\hline $60 \times 42$ & $0.8743+0.0504 i$ \\
$75 \times 42$ & $0.8685+0.0504 i$ \\
$100 \times 42$ & $0.6885+0.0504 i$ \\
$75 \times 42$ & $0.8685+0.0504 i$ \\
$75 \times 46$ & $0.8685+0.0505 i$ \\
$75 \times 50$ & $0.8685+0.0505 i$ \\
\hline
\end{tabular}



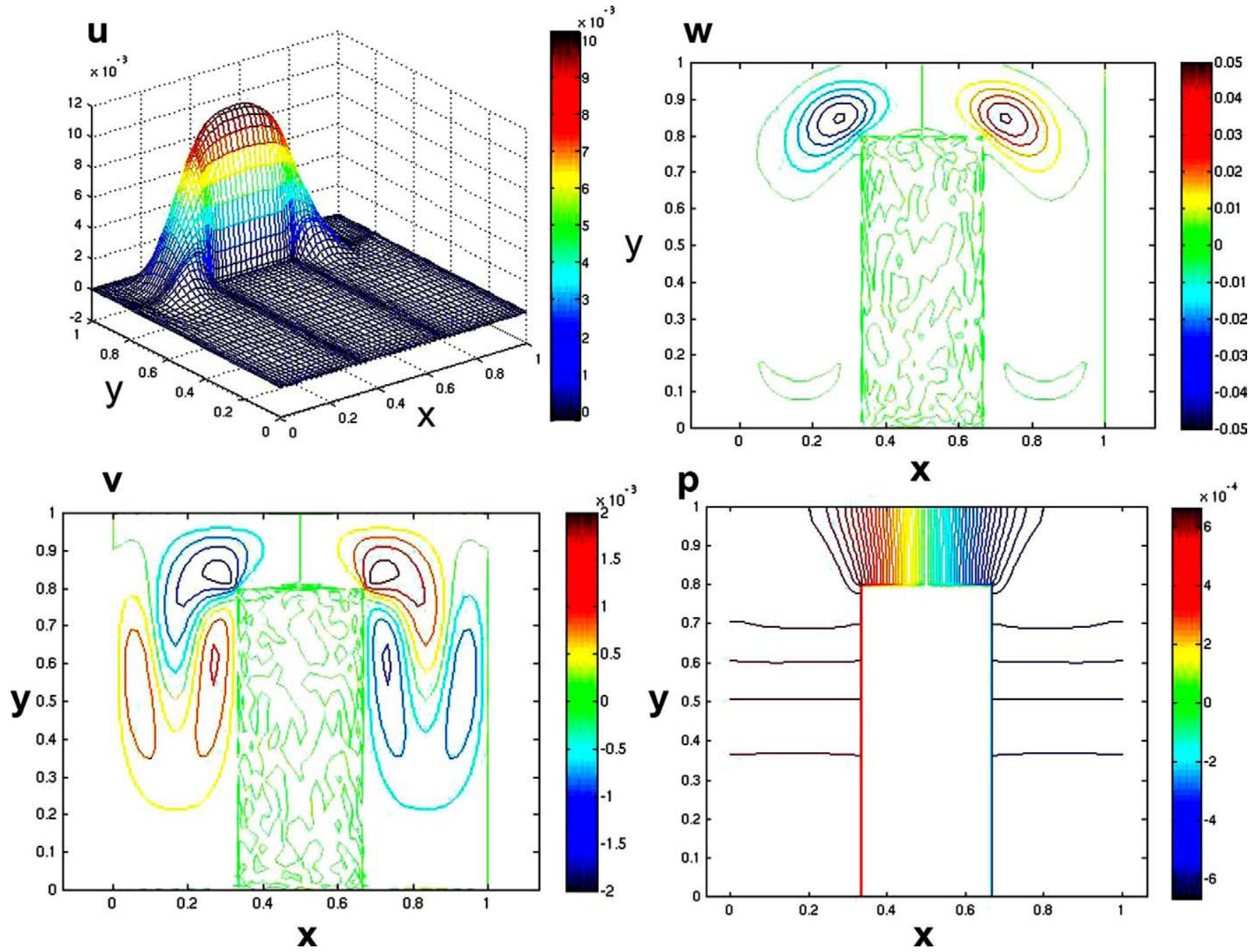

FIG. 5. (Color online) Most unstable eigenmode for $L=1 / 3, H=1 / 5, \operatorname{Re}=50000$, and $\alpha=1.0$. Distribution for the real part velocity components and the pressure.

Extensive numerical studies have been performed, however, for concentric annular channels and pipes. It is well known that linear stability analysis is not able to predict instability for pipe flow (i.e., pipe flow is linearly stable for every value of the Reynolds number and any infinitesimal perturbation), and overpredicts the critical Reynolds number for concentric annular channels and the flow between parallel plates. The reasons for these failures are not entirely understood. Possible explanations are finite amplitude effects, ${ }^{17}$ the nonorthonormality of the Navier-Stokes operator, ${ }^{18}$ spatial effects, or nonlinear effects. ${ }^{19}$

TABLE II. Critical Reynolds number and critical wavenumber as a function of the gap height (the gap length is constant $L=1 / 3$ ).

\begin{tabular}{ccc}
\hline \hline $\mathrm{H}$ & $\mathrm{Re}$ & $\alpha$ \\
\hline 0.4 & 2600 & 2.1 \\
0.2 & 1300 & 2.2 \\
\hline \hline
\end{tabular}

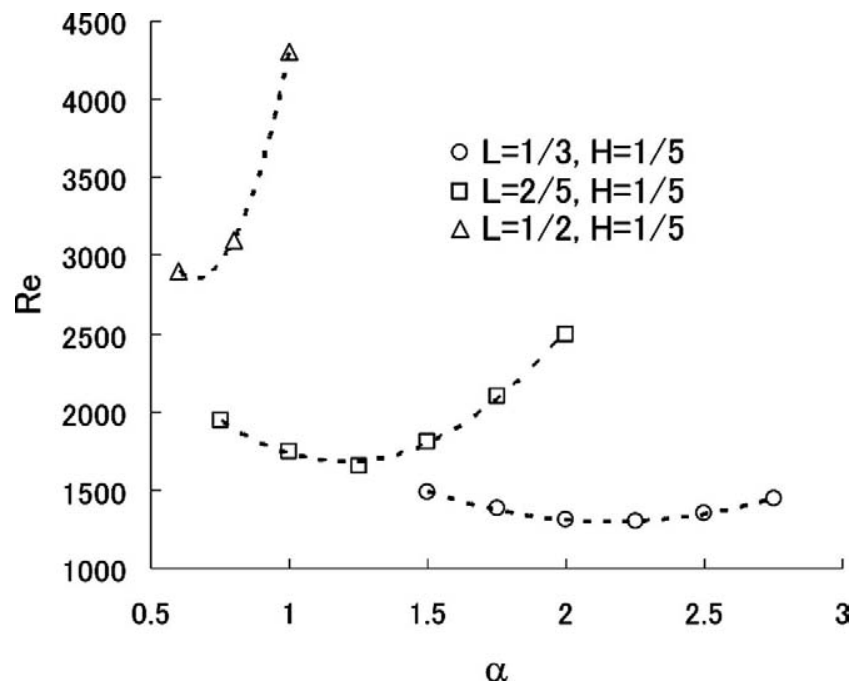

FIG. 6. Marginal stability curves for U-shaped channels and two values of the geometric parameter $L$ ( $H$ is constant). The continuous curves are second order polynomial fits to the computational data. 
Therefore, the objective of the present study cannot be the correct prediction of the instability threshold. It can be, however, a starting point for the analysis of the problem of transition. And it can provide some information regarding the effect of eccentricity and the presence of the narrow gap on the linear stability problem.

The linear stability problem can be solved in the bicylindrical set of coordinates $(\xi, \eta, z)$, where $(\xi, \eta)$ are the bipolar coordinates defined as follows as a function of the Cartesian coordinates $(x, y)$ :

$$
\xi+i \eta=-c \operatorname{coth}^{-1} \frac{x+i y}{2}
$$

and the Cartesian coordinates can therefore be expressed as

$$
\begin{aligned}
& x(\xi, \eta)=c \frac{\sinh \xi}{\cosh \xi-\cos \eta} \\
& y(\xi, \eta)=c \frac{\sin \eta}{\cosh \xi-\cos \eta},
\end{aligned}
$$

where $c$ is a function of the geometric parameters $g=R_{1} / R_{2}$ and $e=d /\left(R_{2}-R_{1}\right)$ (where $d$ is the distance between the axis of the two cylinders):

$$
c=R_{1} \sinh \left[\cosh ^{-1}\left(\frac{g\left(1+e^{2}\right)+1-e^{2}}{2 e g}\right)\right] .
$$

The Jacobian $h$ can then be defined as

$$
h(\xi, \eta)=\frac{c}{\cosh \xi-\cos \eta} .
$$

By simply rewriting the vector form of the Navier-Stokes equation in bicylindrical coordinates, it is possible to obtain

$$
\begin{aligned}
\frac{\partial u}{\partial t}+ & \frac{u}{h} \frac{\partial u}{\partial \xi}+\frac{v}{h} \frac{\partial u}{\partial \eta}+w \frac{\partial u}{\partial z}+u^{2} \sinh \xi-u v \sin \eta \\
= & -\frac{1}{h} \frac{\partial p}{\partial \xi}+\frac{1}{\operatorname{Re}}\left(\frac{1}{h^{2}} \frac{\partial^{2} u}{\partial \xi^{2}}+\frac{1}{h^{2}} \frac{\partial^{2} u}{\partial \eta^{2}}+\frac{\partial^{2} u}{\partial z^{2}}\right. \\
& \left.-\frac{2 \sin \eta}{h} \frac{\partial v}{\partial \xi}+\frac{2 \sinh \xi}{h} \frac{\partial v}{\partial \eta}-\frac{\cosh \xi+\cos \eta}{h} u\right),
\end{aligned}
$$

$$
\begin{aligned}
\frac{\partial v}{\partial t}+ & \frac{u}{h} \frac{\partial v}{\partial \xi}+\frac{v}{h} \frac{\partial v}{\partial \eta}+w \frac{\partial v}{\partial z}-u v \sinh \xi+u^{2} \sin \eta \\
= & -\frac{1}{h} \frac{\partial p}{\partial \eta}+\frac{1}{\operatorname{Re}}\left(\frac{1}{h^{2}} \frac{\partial^{2} v}{\partial \xi^{2}}+\frac{1}{h^{2}} \frac{\partial^{2} v}{\partial \eta^{2}}+\frac{\partial^{2} v}{\partial z^{2}}\right. \\
& \left.+\frac{2 \sin \eta}{h} \frac{\partial u}{\partial \xi}-\frac{2 \sinh \xi}{h} \frac{\partial u}{\partial \eta}-\frac{\cosh \xi+\cos \eta}{h} v\right),
\end{aligned}
$$

$$
\begin{aligned}
\frac{\partial w}{\partial t} & +\frac{u}{h} \frac{\partial w}{\partial \xi}+\frac{v}{h} \frac{\partial w}{\partial \eta}+w \frac{\partial w}{\partial z} \\
& =-\frac{\partial p}{\partial \eta} z+\frac{1}{\operatorname{Re}}\left(\frac{1}{h^{2}} \frac{\partial^{2} w}{\partial \xi^{2}}+\frac{1}{h^{2}} \frac{\partial^{2} w}{\partial \eta^{2}}+\frac{\partial^{2} w}{\partial z^{2}}\right),
\end{aligned}
$$

$\frac{1}{h^{2}}\left(\frac{\partial(h u)}{\partial \xi}+\frac{\partial(h v)}{\partial \eta}\right)+\frac{\partial w}{\partial z}=0$.

The equations for the linear instability problem can be derived by superposing the following infinitesimal disturbance $\left(u^{\prime}, v^{\prime}, w^{\prime}, p^{\prime}\right)$ to the laminar velocity profile $(0,0, W)$ :

$$
\begin{aligned}
u^{\prime} & =i \widetilde{u}(\xi, \eta) \exp [i(\alpha z-\sigma t)], \\
v^{\prime} & =\widetilde{v}(\xi, \eta) \exp [i(\alpha z-\sigma t)], \\
w^{\prime} & =\widetilde{w}(\xi, \eta) \exp [i(\alpha z-\sigma t)], \\
p^{\prime} & =\widetilde{p}(\xi, \eta) \exp [i(\alpha z-\sigma t)] .
\end{aligned}
$$

After linearization, the equations can be rewritten as

$$
\begin{aligned}
& -i \sigma \widetilde{u}=-i W \alpha \tilde{u}+i \frac{1}{h} \frac{\partial \widetilde{p}}{\partial \xi}+\frac{1}{\operatorname{Re}}\left(\frac{1}{h^{2}} \frac{\partial^{2} \tilde{u}}{\partial \xi^{2}}+\frac{1}{h^{2}} \frac{\partial^{2} \tilde{u}}{\partial \eta^{2}}\right. \\
& -\alpha^{2} \widetilde{u}+i \frac{2 \sin \eta}{h} \frac{\partial \widetilde{v}}{\partial \xi}-i \frac{2 \sinh \xi}{h} \frac{\partial \widetilde{v}}{\partial \eta} \\
& \left.-\frac{\cosh \xi+\cos \eta}{h} \widetilde{u}\right) \text {, } \\
& -i \sigma \widetilde{v}=-i W \alpha \widetilde{v}-\frac{1}{h} \frac{\partial \widetilde{p}}{\partial \eta}+\frac{1}{\operatorname{Re}}\left(\frac{1}{h^{2}} \frac{\partial^{2} \widetilde{v}}{\partial \xi^{2}}+\frac{1}{h^{2}} \frac{\partial^{2} \widetilde{v}}{\partial \eta^{2}}\right. \\
& -\alpha^{2} \widetilde{v}+i \frac{2 \sin \eta}{h} \frac{\partial \widetilde{u}}{\partial \xi}-i \frac{2 \sinh \xi}{h} \frac{\partial \widetilde{u}}{\partial \eta} \\
& \left.-\frac{\cosh \xi+\cos \eta}{h} \tilde{v}\right) \text {, } \\
& -i \sigma \widetilde{w}=-i W \alpha \widetilde{w}-i \tilde{u} \frac{\partial W}{\partial \xi}-\widetilde{v} \frac{\partial W}{\partial \eta}-i \alpha \tilde{p} \\
& +\frac{1}{\operatorname{Re}}\left(\frac{1}{h^{2}} \frac{\partial^{2} \widetilde{w}}{\partial \xi^{2}}+\frac{1}{h^{2}} \frac{\partial^{2} \widetilde{w}}{\partial \eta^{2}}-\alpha^{2} \widetilde{w}\right), \\
& \frac{i}{h^{2}}\left(\frac{\partial(h \widetilde{u})}{\partial \xi}-i \frac{\partial(h \widetilde{v})}{\partial \eta}\right)+i \alpha \widetilde{w}=0 .
\end{aligned}
$$

For each pair of values $(\operatorname{Re}, \alpha)$ the equations become a complex eigenfunction-eigenvalue problem in $(\widetilde{u}, \widetilde{v}, \widetilde{w}, \widetilde{p})$ and $\sigma$. The laminar distribution $W$ can be obtained by solving a laminar problem similar to Eq. (4); however, an analytical solution has been obtained by Snyder and Goldstein ${ }^{20}$ for $W$ :

$$
\begin{aligned}
W(\eta, \xi)= & F+E \eta-\frac{1}{2} \frac{\cosh (\eta)}{\sinh (\eta)} \\
& +\sum_{n}\left[A_{n} e^{n \eta}+\left(B_{n}-\frac{\cosh (\eta)}{\sinh (\eta)}\right) e^{-n \eta}\right] \cos (n \xi),
\end{aligned}
$$

where $F, E, A$, and $B$ are coefficients that depend on the geometry (Snyder and Goldstein ${ }^{20}$ ). As an additional confirmation, it has then been verified a posteriori that the expression given by Eq. (15) is a solution of the laminar problem.

In the present case a nonstaggered approach has been applied for the pressure. The validity of the approach has 
TABLE III. Most unstable eigenvalues for the implemented 1D polar code.

\begin{tabular}{ccc}
\hline \hline Rank & Present code 1D polar code & Orszag (1971) \\
\hline 1 & $0.237543+0.00375 i$ & $0.23752649+0.00373967 i$ \\
2 & $0.9646-0.035617 i$ & $0.96463092-0.03561728 i$ \\
3 & $0.9646-0.0351867 i$ & $0.96464251-0.03518658 i$ \\
4 & $0.27715-0.05098 i$ & $0.27720434-0.05089873 i$ \\
5 & $0.9363165-0.0632 i$ & $0.93631654-0.06320150 i$ \\
6 & $0.9363521-0.063252 i$ & $0.93635178-0.06325157 i$ \\
7 & $0.907983-0.0912221 i$ & $0.90798305-0.09122274 i$ \\
8 & $0.90806-0.091314 i$ & $0.90805633-0.09131286 i$ \\
9 & $0.8796271-0.11923 i$ & $0.87962729-0.11923285 i$ \\
10 & $0.8797565-0.119372 i$ & $0.87975570-0.11937073 i$ \\
\hline \hline
\end{tabular}

been addressed by Khorrami and Malik. ${ }^{21}$ The following boundary condition for the pressure is assumed to be respected at the wall:

$$
i \frac{1}{h} \frac{\partial \tilde{p}}{\partial \xi}=-\frac{1}{\operatorname{Re}}\left(\frac{1}{h^{2}} \frac{\partial^{2} \tilde{u}}{\partial \xi^{2}}+i \frac{2 \sin \eta}{h} \frac{\partial \tilde{v}}{\partial \xi}\right),
$$

while nonslip boundary conditions are considered for the velocity components. Periodic boundary conditions are implemented in the spanwise direction.

The derivates in the inhomogeneous spatial directions $(\xi, \eta)$, can be discretized by using spectral collocation techniques. An interesting peculiarity of this choice, however, is that Chebyshev polynomials are not necessarily the optimal choice for the wall normal direction. In fact, it can be proved that in the limit $e \rightarrow 0$ the set of collocation points obtained in this case do not coincide with the collocation points for the radial direction associated to Chebyshev polynomials in a concentric channel.

In the present case, Chebyshev polynomials and Fourier functions have been selected as basis functions for the application of the spectral collocation methodology. The problem then reduces to a linear system of the type already described in Eq. (5) which can be solved using the same techniques described in Sec. II A.

\section{B. Validation}

Since there are no studies available on the Pouseille flow between eccentric channels, the methodology has been tested on the limit case of the concentric channel $(e \rightarrow 0)$.

In order to provide an extensive validation, the results of the bicylindrical code have been tested against the results obtained for two-dimensional (2D) and one-dimensional (1D) cylindrical codes. The codes have been written following the example of Khorrami and Malik. ${ }^{21}$ The accuracy of the cylindrical codes has been verified by benchmarking toward results published in the literature.

In particular, in the limit $g \rightarrow 1$, the spectrum of the concentric channel is bound to be a good approximation of the spectrum of channel flow (the curvature effect of the inner cylinder tends to be negligible). This intuitive result has been confirmed by early works, ${ }^{22}$ and here. In Table III a comparison is available between the most unstable modes for channel flow (computed by Orszag ${ }^{23}$ ) and the ones obtained for
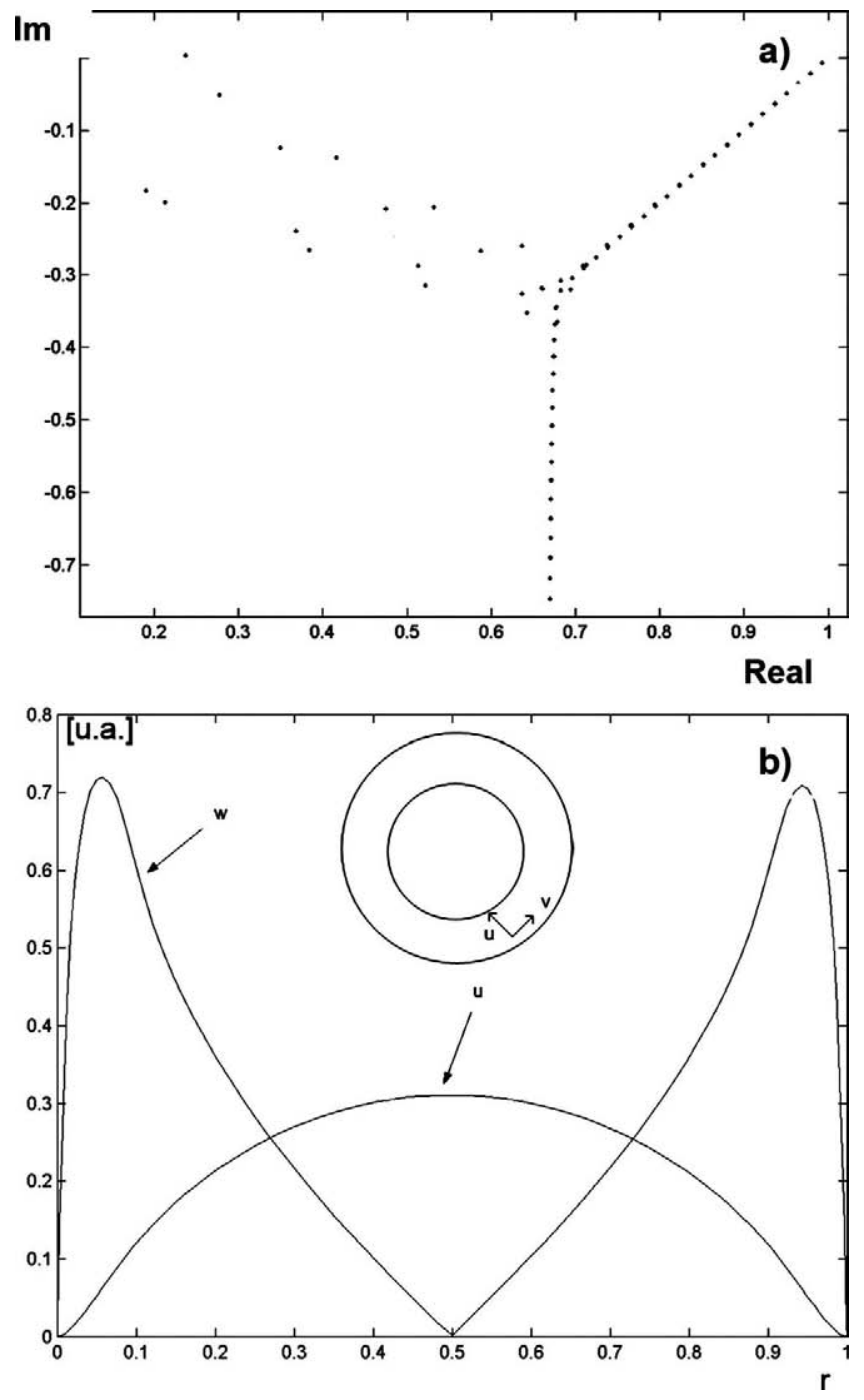

FIG. 7. 1D polar code results, eigenvalue spectrum (a) and most unstable eigenfunction (b).

the flow in a concentric annular channel with $g=0.99$. The specific code employed is $1 \mathrm{D}$ and only axial-symmetric disturbances are considered. The Reynolds number is equal to 10000 and the wavenumber $\alpha$ is equal to 1 . The discretization employed includes 64 nodes in the radial direction. Improved agreement is obtained if an increasing number of collocation nodes is employed. In Fig. 7, the eigenvalue spectrum for the same condition is shown. It is possible to notice that the S-family is in very good approximation located near $R e=2 / 3$, as predicted by the theory of linear stability for channel flow. Figure 7(b) also shows the radial distributions for the most unstable eigenfunction. The agreement with previous works is overall good.

The cylindrical code thus validated has been employed as a benchmarking tool for the bicylindrical code. In Fig. 8 it is possible to observe a comparison between the data obtained for the three algorithms employed for a case with $g=0.7$ and $\operatorname{Re}=5000$.

It is possible to notice that the $2 \mathrm{D}$ codes produce very similar results; differences between the two are related to the choice of Chebyshev polynomial for the discretization of the 


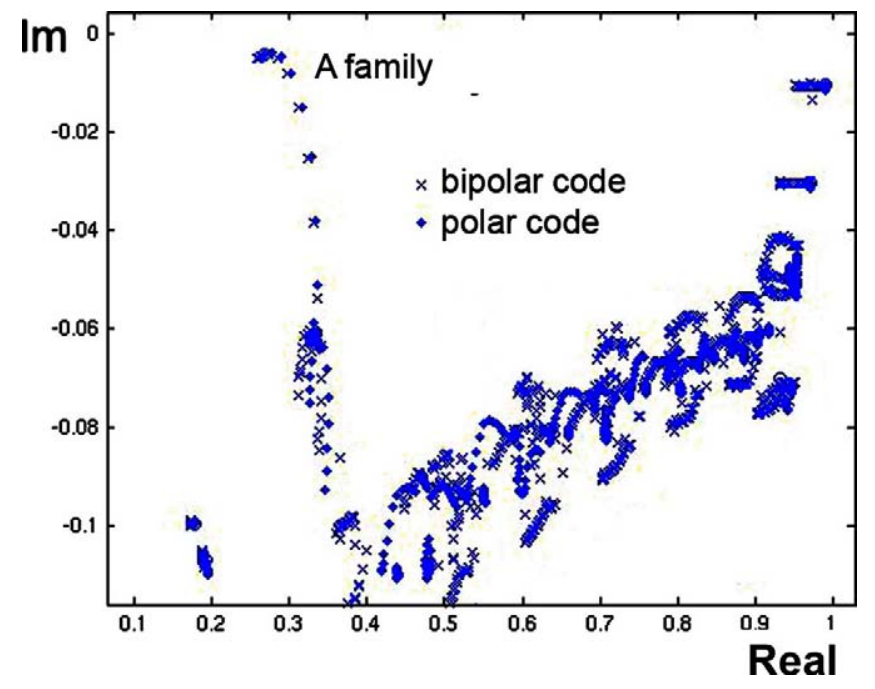

FIG. 8. (Color online) Comparison between 2D polar code and bicylindrical code for $e=0.01, g=0.7$, and $\mathrm{Re}=5000$.

wall normal direction in bipolar coordinates (it is not opti$\mathrm{mal}$ ), and concern only higher order eigenvalues ( $\mathrm{S}$ family). Moreover, the results of the 2D codes include the results obtained by the $1 \mathrm{D}$ code.

\section{Results}

All results obtained in this section have been obtained with a grid resolution of $64 \times 64$. The high resolution in the spanwise direction is justified by the inhomogeneous character of the cross section.

\section{Low eccentricity}

The eigenvalue spectrum of the linear stability problem for eccentric annular channels does not differ substantially from the spectrum for concentric channels at low eccentricity. The reduction in symmetry, however, leads to a less regular spectrum (i.e., in high symmetrical systems several of the eigenvalues have multiplicity bigger than one, while in lowsymmetrical systems high-multiplicity eigenvalues tend to be

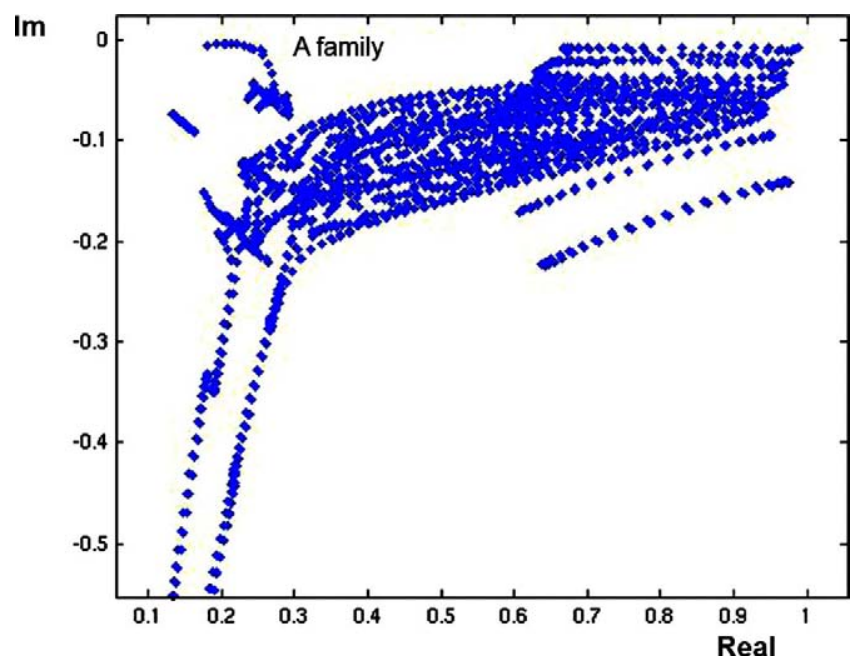

FIG. 9. (Color online) Eigenvalue spectrum, $e=0.1, g=0.7$, and $\mathrm{Re}=7000$.
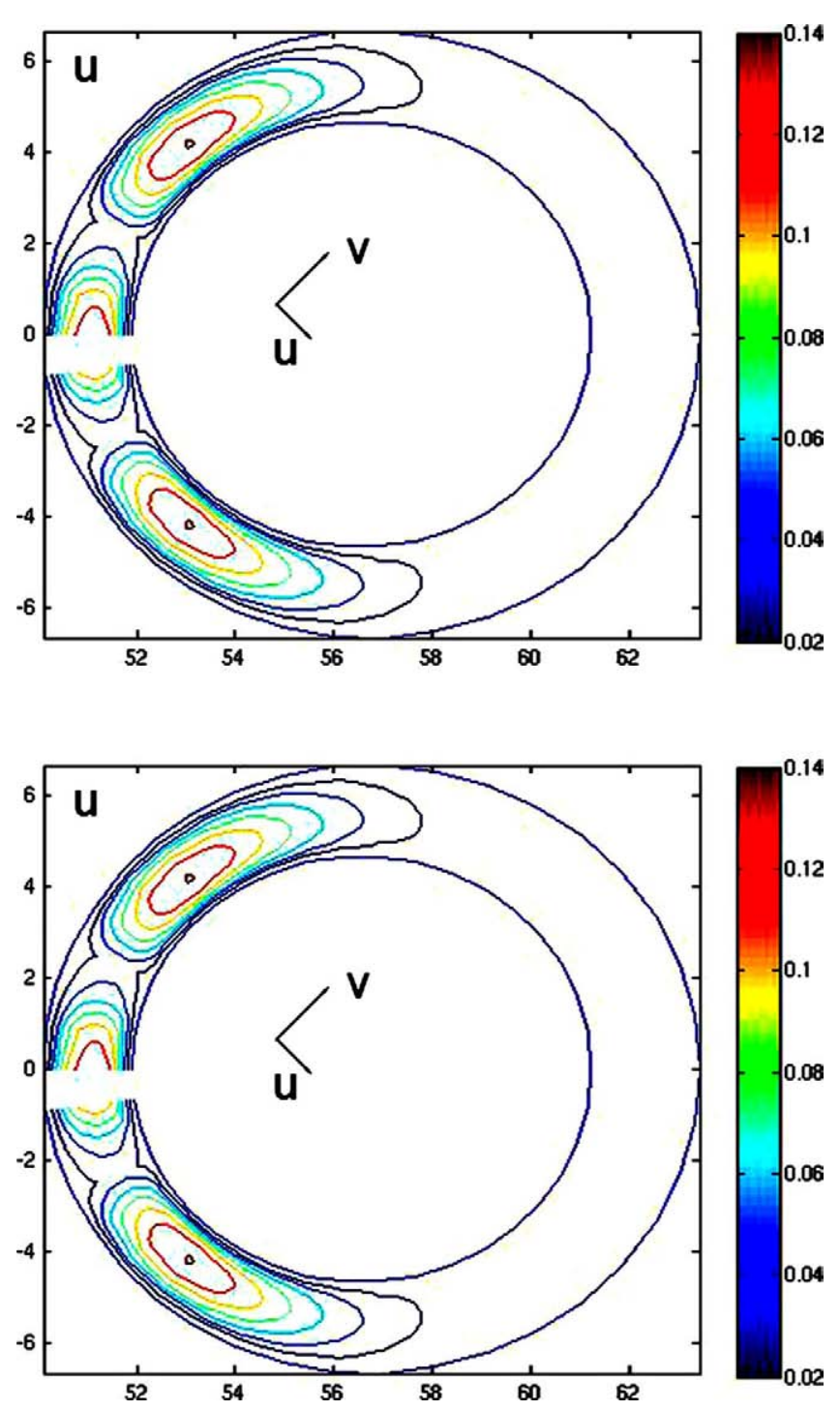

FIG. 10. (Color online) Most unstable eigenmode for $e=0.1, g=0.7$, and $\operatorname{Re}=7000$. Velocity distribution for the velocity components $u$ and $w$.

less common), and the branches of modes clearly discernible for concentric channels mix in a nontrivial manner. The most unstable modes, however, are still given by the A-family, at least for low eccentricity. Eccentric annular channels are more stable than concentric channels for the same values of $g$ and Re. Figure 9 shows a typical eigenvalue spectrum for the flow in eccentric annular channels $(g=0.7, e=0.1, \alpha$ $=1.0$ ). The most unstable mode for the same case is shown in Fig. 10 and it is comparable with the most unstable eigenmode for a concentric channel at $g=0.7$ [Fig. 7(b)].

In the eigenvalue spectrum of the flow in eccentric channel, there is a particular set of modes that is increasingly unstable as the eccentricity increases. The nature of these modes can be associated with the presence of a spanwise variation for the mean velocity profile. Henningson ${ }^{24}$ showed that if a spanwise variation [of the type shown in Eq. (17)] is added to the mean flow the eigenvalue spectrum of the Pouseille flow in concentric channels and pipe channels might contain additional unstable modes. 
Im

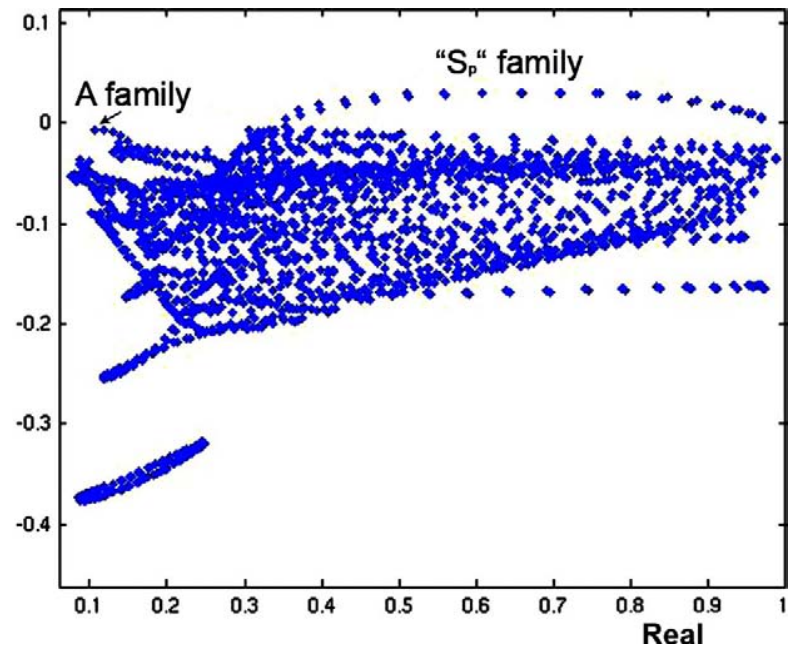

FIG. 11. (Color online) Eigenvalue spectrum for $g=0.7$ and $\mathrm{Re}=7000$ (concentric annulus with mean spanwise variation).

A numerical experiment has been conducted on a concentric annular channel, with $g=0.7, \alpha=1$, and $\operatorname{Re}=5000$ the results are reported in Fig. 11. It is possible to notice that for the same Reynolds number $(\operatorname{Re}=5000)$ is dramatically dif- ferent if compared to Fig. 8. In particular, an entire new branch is introduced that will be in the following addressed as "Sp" branch or family. The laminar profile studied corresponds to the following distribution:

$$
W=\frac{1-r^{2}+r_{m}^{2} \ln \left(r^{2}\right)}{1-r_{m}^{2}+r_{m}^{2} \ln \left(r_{m}^{2}\right)}+a \cos (k \theta),
$$

where $r_{m}$ is the radial position of the velocity maximum and $a$ and $k$ are parameters.

A three-dimensional plot of the perturbation added to the streamwise velocity profile as well as the most unstable perturbation (member of the Sp-family) are shown in Fig. 12.

The same family of modes, or a very similar family of modes, is present in eccentric channels, at low eccentricity their presence is masked by other modes, since they do not appear clearly as a family. Moreover they do not play an essential role in the linear stability problem, while at higher eccentricity they become the main cause of instability.

Figure 13(a) shows the curves of marginal stability computed by considering the A family only. The problem has been given by Eq. (10) repeatedly for hundreds of values of $\alpha$ and $\operatorname{Re}$ through the Arnoldi algorithm. It is possible to notice that, at low values of eccentricity, the higher the ec-
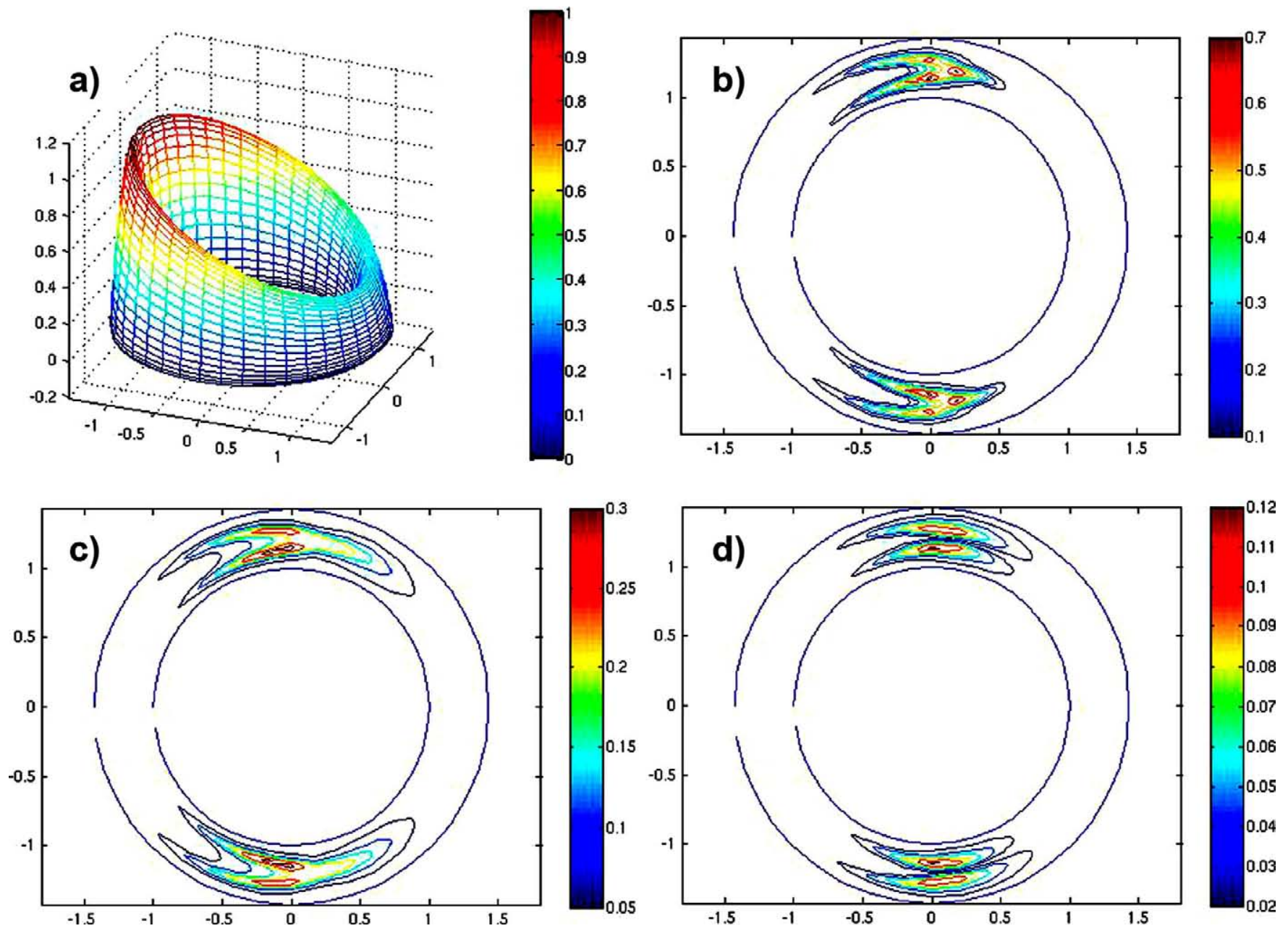

FIG. 12. (Color online) Most unstable eigenmode for $g=0.7$ and $\mathrm{Re}=5000$ (concentric annulus with mean spanwise variation): laminar streamwise distribution (a); streamwise velocity (b), and components $v$ (c) and $u$ (d). 

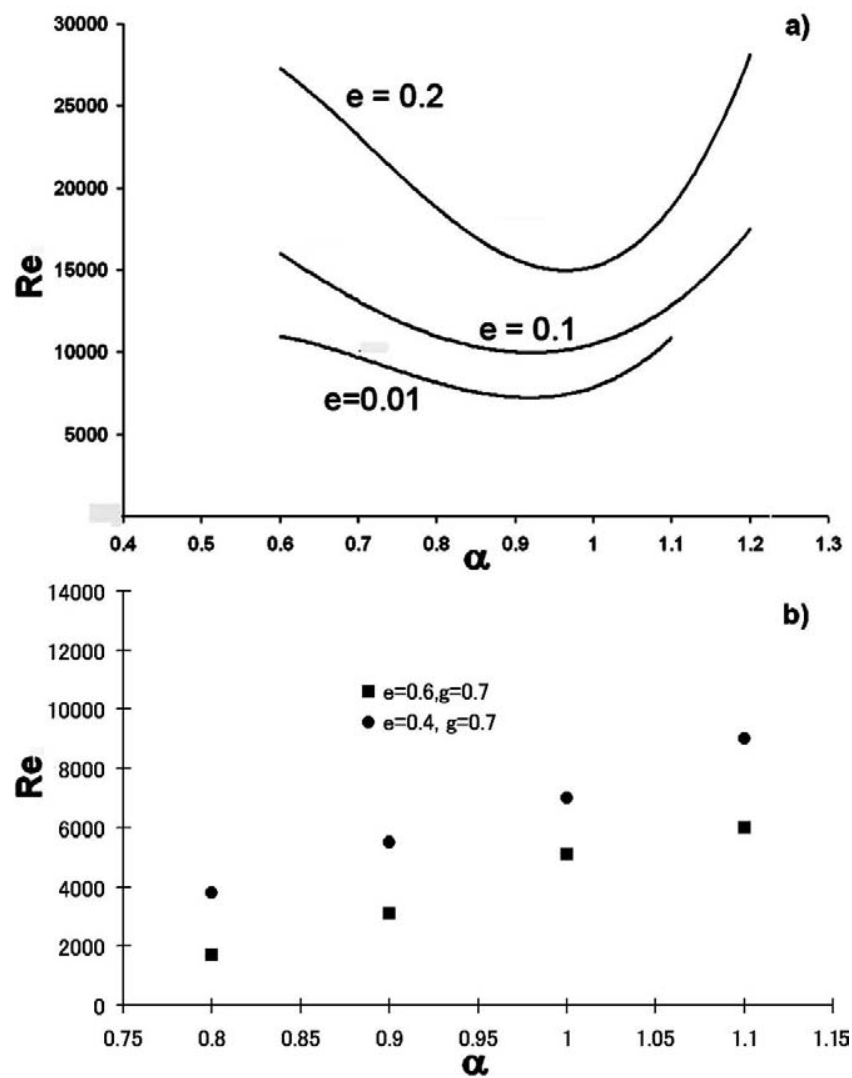

FIG. 13. Portion of the curves of marginal stability at different values of eccentricity for $g=0.7$. Low eccentricity (a) and high eccentricity (b).

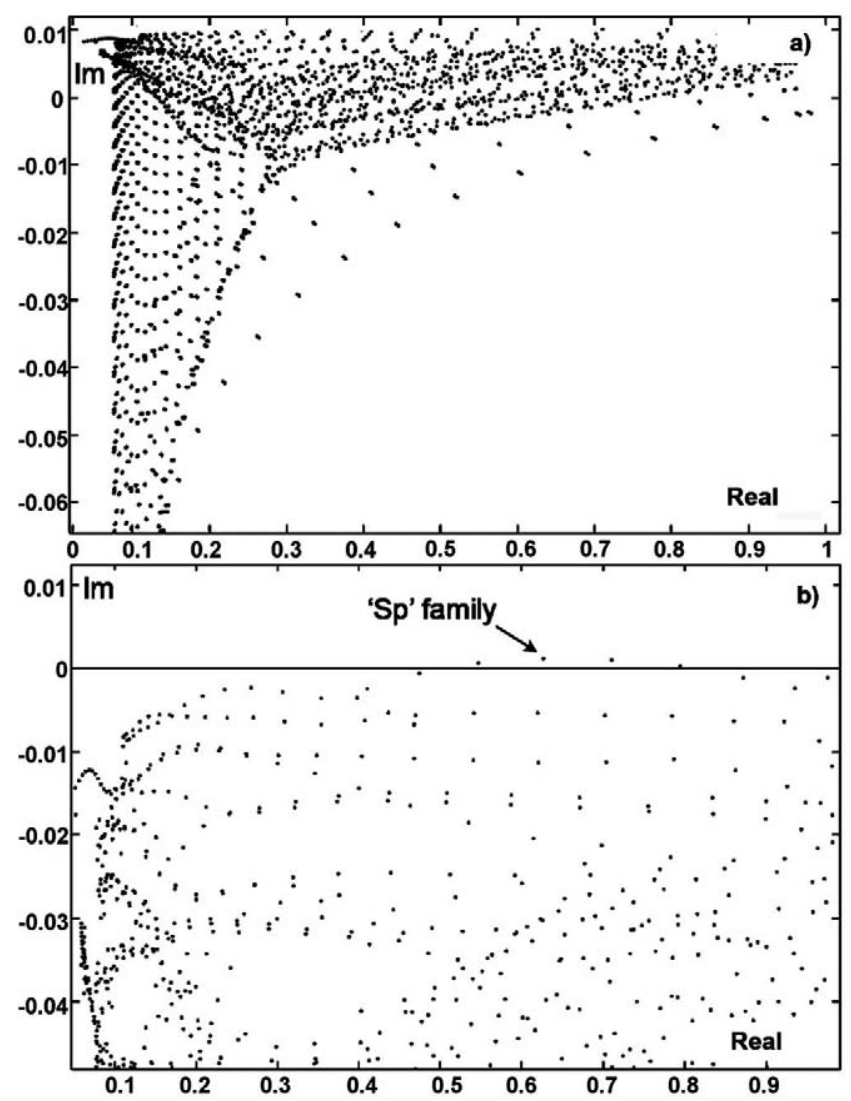

FIG. 14. Eigenvalue spectrum (a) for $e=0.5, g=0.7$, and $\mathrm{Re}=7000$; with detail of the $\operatorname{Im}=0$ region (b).
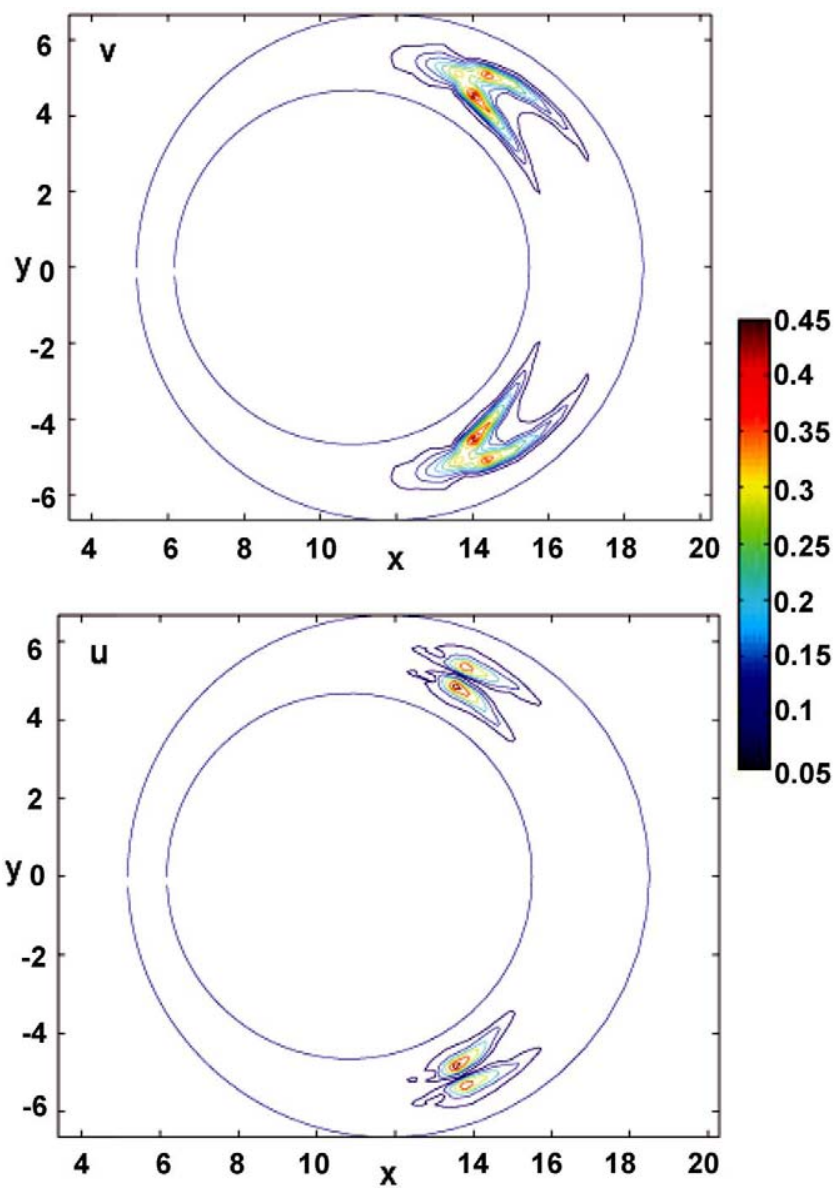

FIG. 15. (Color online) Most unstable eigenmode for $e=0.5, g=0.7$, Re $=7000$. Components $u$ and $v$ of the velocity.

centricity the more stable is the flow. In fact, the concentric channel is the most unstable configuration if the other parameters $(g, \mathrm{Re})$ are considered constant. Therefore at low eccentricity (e.g., $e \leq 0.3$ for $g=0.7$ ), the main effect of an increase in eccentricity is a drift of the curve of marginal stability toward higher $\operatorname{Re}$ and $\alpha$.

\section{High eccentricity}

The trend shown in Fig. 11(a), shows clearly that the A-family is progressively more stable as eccentricity increases. However, the A-family is not the only set of modes able to generate unstable eigenmodes at high eccentricity.

Figure 14 shows the eigenvalue spectrum for $g=0.7$, $e=0.5$, and $\operatorname{Re}=7000$. From extrapolation of the results presented in Fig. 13, it is evident that the flow should be stable to infinitesimal perturbation at this Reynolds number (it should be stable even for $e=0.0$ ). However, this is not case. Several eigenmodes appear to be linearly unstable, and they can be recognized as part of the Sp family identified for concentric channels with spanwise variation. Figure 15 shows a contour plot of the absolute value of the velocity components of the most unstable perturbation. They appear to be very similar to the results of Fig. 12. Therefore, it may be concluded that eccentric channels at sufficient high values 
TABLE IV. Most unstable eigenvalues for $g=0.7, e=0.5, \operatorname{Re}=7000$, and $\alpha=1$.

\begin{tabular}{ccc}
\hline \hline Grid & Most unstable & Second most unstable \\
\hline $45 \times 80$ & $0.6184+0.00136 i$ & $0.712+0.00125 i$ \\
$45 \times 90$ & $0.6184+0.00134 i$ & $0.712+0.00124 i$ \\
$64 \times 64$ & $0.6185+0.00134 i$ & $0.711+0.00124 i$ \\
$64 \times 80$ & $0.6184+0.00136 i$ & $0.712+0.00125 i$ \\
\hline \hline
\end{tabular}

of eccentricity are linearly unstable at lower values than their concentric channel counterparts. Moreover the instability can be associated to the spanwise variation of the streamwise velocity component $W$.
An additional numerical test has been conducted in order to verify this important result. In Table IV the two most unstable eigenvalues for the case $g=0.7, e=0.5, \operatorname{Re}=7000$, and $\alpha=1$ are shown for four different grids. It is demonstrated that the eigenvalue spectrum is close to convergence for the four grids and a resolution of $64 \times 64$ is deemed to be acceptable. It has to be noted that an exponential variation of the mesh in the spanwise direction has been adopted in order to achieve a more uniform grid.

The effect of eccentricity on the eigenvalue spectrum is shown in Fig. 16(a) where the value of the most unstable eigenvalue is shown as a function of $e$. The critical eccentricity depends also upon the value of $g$ [Fig. 16(a)]. The laminar flow is increasingly unstable as the Reynolds num-
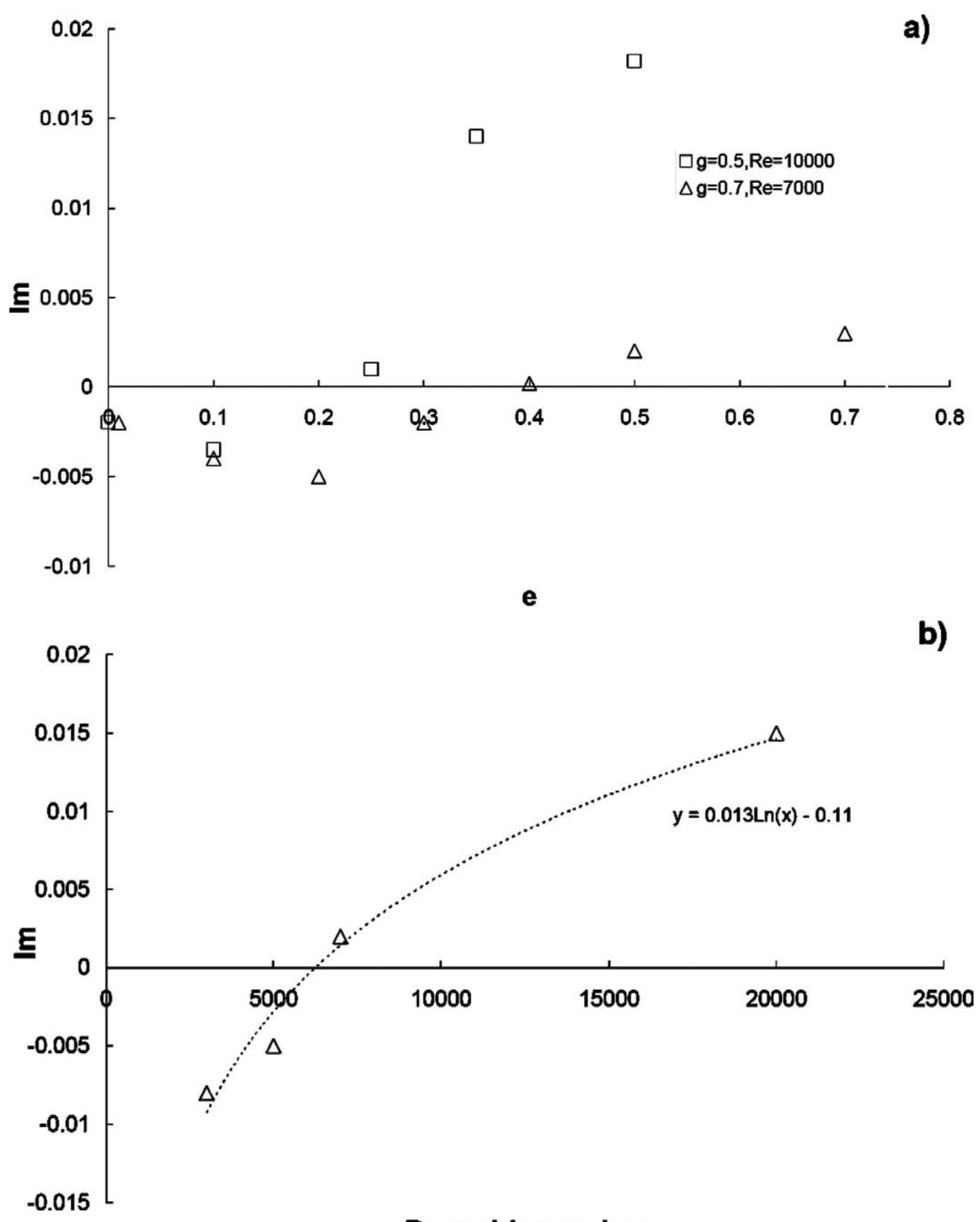

\section{Reynolds number}

FIG. 16. Most unstable eigenvalue as a function of eccentricity (a), and as a function of the Reynolds number (b) at $g=0.7$ and $e=0.5$ with a logarithmic fit. For both graphs $\alpha=1.0$. 

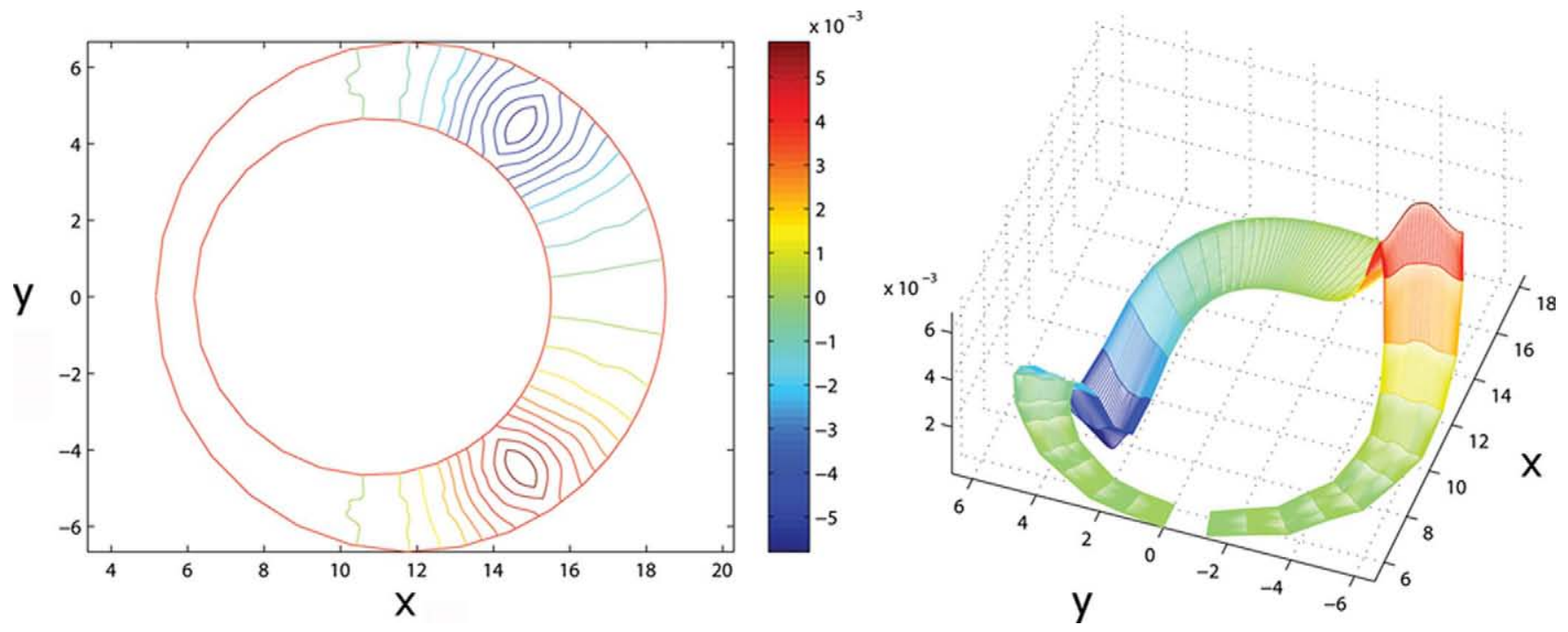

FIG. 17. (Color online) Most unstable eigenmode distribution for the pressure, $e=0.5, g=0.7$, and $\operatorname{Re}=7000$.

ber increases. It appears that a logarithmic law well approximates the dependency of the imaginary part of the most unstable mode of the Sp family upon the Reynolds number [Fig. 16(b), the data refer to $g=0.7, e=0.5$, and $\alpha=1$ ]. As a consequence of the presence of these unstable modes, the curves of marginal stability shift toward lower Reynolds numbers [Fig. 13(b)]. The critical Reynolds number appears to be lower than the concentric case, which is at odds with what observed experimentally by Gosset and Tavoularis. ${ }^{1}$

In fact, Gosset and Tavoularis ${ }^{1}$ found that for a similar geometry, an increased value of eccentricity leads to more stable laminar flows. The calculations performed seem to confirm this observation for small values of the eccentricity. However, the critical Reynolds number predicted by the linear theory [Fig. 13(a)] is significantly higher than the experiment value for the concentric channel. This disagreement is not surprising since linear stability analysis is not usually able to reproduce the stability properties of laminar flows in pipes and concentric channels. Full nonlinear transient growth studies are necessary to reproduce accurately the process of transition in these geometries.

For higher values of the eccentricity [Fig. 13(b)], the critical Reynolds number is sensibly lower than the concentric case and it appears to be closer to experimental values for pipe flow. ${ }^{17}$ This observation suggests that, while at lower eccentricity the mechanism of transition cannot be explained through the linear theory, the present analysis may lead to a more accurate prediction of the stability threshold at high eccentricity. It also reinforces the idea that the mechanism of transition in this geometry is radically different from concentric pipes.

In conclusion, despite the limitations of the present approach, a few significant conclusions can be drawn from the present calculations:

(1) At low values of eccentricity, eccentricity plays a stabilizing role, since eccentric channels have more stable A-family modes than concentric channels;

(2) an additional family of modes is present in eccentric channels, related to the spanwise variation of the streamwise velocity field;

(3) this additional family of modes induces instability at lower values of the Reynolds number than the A-branch for high values of eccentricity $(e \geq 0.3)$.

Thus the usual process of transition in concentric channels and pipes is stabilized at higher eccentricity, but an additional process of instability comes into play. The most unstable perturbation associated with this mechanism develops in the wider gap region and is related to the spanwise variation of the streamwise velocity profile $W$. The connection between the family of modes presented in this section and the mechanism of instability discovered in Sec. II C can be found when observing the pressure distribution for the most unstable mode. In fact, the Sp-family modes are characterized by an antisymmetric pressure wave of the type already discussed in Sec. II C (Fig. 17 shows the pressure distribution for the most unstable mode, $e=0.5, g=0.7$, and Re $=7000$ ). The difference between the shapes of the waves in the two cases might be related to the shape of the cross section (i.e., in eccentric channels there are actually two gaps: a wide gap and a narrow gap; while in U-shaped channels only a narrow gap can be identified). Both geometries are characterized by a single symmetry axis.

\section{CONCLUSIONS}

The global linear stability problem for two geometry classes containing a narrow gap has been studied through spectral collocation. Compared to the results typical of channel flow, concentric annular channel flow or pipe flow, the results show that an additional mechanism of instability is present.

In particular, in eccentric channels, a new branch of modes is introduced that can be recognized as to be a consequence of the spanwise variation of the laminar velocity profile. The effect is significant at high eccentricity (the 
range examined goes from $e=0.01$ to 0.8 ) and depends overall upon the internal to external diameter ratio and the eccentricity. The most unstable eigenmodes are characterized by spanwise variation in the wide gap region and the propagation of an asymmetric pressure wave in the streamwise direction. This mechanism of instability might justify the onset of oscillations in the wide gap region observed by Gosset and Tavoularis ${ }^{1}$ for a similar geometry. However a full nonlinear transient growth study is necessary to clarify how these initials oscillations develop into coherent structures in the narrow gap region at higher Reynolds numbers.

For the U-shaped channel case, rather than a family of modes a single unstable mode has been found, at least for the calculations performed here. As in the case of eccentric channel the most unstable mode of turbulence is characterized by the propagation in the streamwise direction of an asymmetric pressure wave. The velocity distribution for the unstable mode presents important similarities with most energetic mode of turbulence observed through a POD (proper orthogonal decomposition) of the flow field performed on a LES (large eddy simulation) database at a $\mathrm{Re}=3000{ }^{14}$ This suggests that

(1) the laminar velocity profile in U-shaped channels go through a process of linear instability and

(2) the most unstable mode dominates the turbulence structure at higher Reynolds numbers.

The relationship between coherent structures and instability modes is further discussed in the works of Uhlmann and co-workers. ${ }^{25,26}$

Future work will include a transient growth study of the most unstable perturbation for eccentric channel and U-shaped channels to clarify their role in the laminarturbulent transition in these geometries. In particular, it will be investigated if such modes develop in the vortex street observed by Guellouz and Tavoularis. ${ }^{4}$

${ }^{1}$ A. Gosset and S. Tavoularis, "Laminar flow instability in a rectangular channel with a cylindrical core," Phys. Fluids 18, 044108 (2006).

${ }^{2} \mathrm{~T}$. Tatsumi and T. Yoshimura, "Stability of the laminar flow in a rectangular duct," J. Fluid Mech. 212, 437 (1990).

${ }^{3}$ C. Canuto, M. Y. Hussaini, A. Quarteroni, and T. A. Zang, Spectral Methods in Fluid Mechanics (Springer-Verlag, Berlin, 1988).

${ }^{4}$ M. S. Guellouz and S. Tavoularis, "The structure of turbulent flow in a rectangular channel containing a cylindrical rod-Part I: Reynolds averaged experiments," Exp. Therm. Fluid Sci. 29, 59 (2000).

${ }^{5}$ J. D. Hooper and K. Rehme, "Large-scale structural effect in developed turbulent flows through closely-spaced rod arrays," J. Fluid Mech. 145, 305 (1984).

${ }^{6}$ E. Merzari and H. Ninokata, "Anisotropy and coherent structures for the flow in annular channels," Flow, Turbul. Combust. (to be published).

${ }^{7}$ A. Lexmond, T. Rudde, and M. Van der Haagen, "Visualization of the vortex street and characterization of the cross flow in the gap between two subchannels," Proceedings of NURETH-11, Avignon, France, 2005.

${ }^{8}$ M. Biemuller, L. Meyer, and K. Rehme, "Large eddy simulation and measurement of the structure of turbulence in two rectangular channels connected by a gap," in Engineering Turbulence Modelling and Experiments: Proceedings of the Third International Symposium, Crete, Greece, 27-29 May 1996 (Elsevier, Amsterdam, 1996), Vol. 3, p. 249.

${ }^{9}$ L. Meyer and K. Rehme, "Large-scale turbulence phenomena in compound rectangular channels," Exp. Therm. Fluid Sci. 8, 286 (1994).

${ }^{10}$ J. W. Demmel and B. Kagstrom, "Computing stable eigendecompositions of matrix pencils," Linear Algebr. Appl. 88-89, 139 (1987).

${ }^{11}$ R. B. Lehoucq and D. C. Sorensen, "Deflation techniques for an implicitly re-started Arnoldi iteration," SIAM J. Matrix Anal. Appl. 17, 789 (1996).

${ }^{12} \mathrm{~V}$. Theofilis, P. W. Duck, and J. Owen, "Viscous linear stability analysis of rectangular duct and cavity flows," J. Fluid Mech. 505, 249 (2004).

${ }^{13}$ A. Jennings, J. Halliday, and M. J. Cole, "Solution of linear eigenvalue problems containing singular matrices," J. Inst. Math. Appl. 22, 401 (1978).

${ }^{14}$ E. Merzari, E. Baglietto, and H. Ninokata, "Numerical simulation of the flow in tight-lattice fuel bundles," Nucl. Eng. Des. 238, 1703 (2008).

${ }^{15}$ G. I. Taylor, "Stability of a viscous liquid contained between two rotating cylinders," Philos. Trans. R. Soc. London, Ser. A 223, 189 (1923).

${ }^{16}$ R. C. Diprima and J. T. Stuart, "Non-local effect on the stability of flow between eccentric rotating cylinders," J. Fluid Mech. 54, 393 (1972).

${ }^{17} \mathrm{P}$. Drazin and W. Reid, Hydrodynamic Stability (Cambridge University Press, Cambridge, 1981).

${ }^{18}$ L. Trefethen, A. E. Trefethen, S. C. Reddy, and T. Driscoll, "Hydrodynamic stability without eigenvalues," Science 261, 578 (1993).

${ }^{19}$ P. Schmid and D. S. Henningson, Stability and Transition in Shear Flows (Springer, New York, 2001).

${ }^{20}$ W. T. Snyder and G. A. Goldstein, "An analysis of fully developed laminar flow in an eccentric annulus," AIChE J. 11, 462 (1965).

${ }^{21}$ M. R. Khorrami and M. R. Malik, "Application of spectral collocation techniques on the stability of swirling flows," J. Comput. Phys. 81, 206 (1989).

${ }^{22}$ J. E. Mott and D. D. Joseph, "Stability of parallel flow between concentric cylinders," Phys. Fluids 11, 2065 (1968).

${ }^{23}$ S. A. Orszag, "Accurate solution of the Orr-Sommersfeld stability equation," J. Fluid Mech. 50, 689 (1971).

${ }^{24}$ D. S. Henningson, "Stability of parallel inviscid shear flow with mean spanwise variation," FFA Technical Report No. TN 1987-57, 1987.

${ }^{25}$ M. Uhlmann, A. Pinelli, G. Kawahara, and A. Sekimoto, "Marginally turbulent flow in a square duct," J. Fluid Mech. 588, 153 (2007).

${ }^{26} \mathrm{M}$. Uhlmann and M. Nagata, "Linear stability of flow in an internally heated rectangular duct," J. Fluid Mech. 551, 387 (2006). 